\title{
663.
}

\section{FURTHER INVESTIGATIONS ON THE DOUBLE 9-FUNCTIONS.}

[From the Journal für die reine und angewandte Mathematik (Crelle), t. LxxxIII. (1877), pp. $220-233$.]

I CONSIDER six letters

$$
a, b, \quad c, d, e, f
$$

a duad $a b$ not containing $f$ may be completed into the triad $a b f$, and then into the double triad $a b f$.cde; there are in all ten double triads, represented by the duads

$$
a b, a c, a d, a e, b c, b d, b e, c d, c e, d e,
$$

and the whole number of letters and of double trizds is $=16$.

Taking $x, x^{\prime}$ as variables, I form sixteen functions; viz. these are

$$
\begin{aligned}
& {[a]=a-x \cdot a-x^{\prime},} \\
& {[a b]=\frac{1}{\left(x-x^{\prime}\right)^{2}}\left\{\sqrt{\begin{array}{c}
a-x \cdot b-x \cdot f-x \\
c-x^{\prime} \cdot d-x^{\prime} \cdot e-x^{\prime}
\end{array}} \pm \sqrt{\begin{array}{c}
a-x^{\prime} \cdot b-x^{\prime} \cdot f-x^{\prime} \\
c-x \cdot d-x \cdot e-x
\end{array}}\right\}^{2},}
\end{aligned}
$$

where the function under each radical sign is the product of six factors, the arrangement in two lines being for convenience only: the sign \pm has the same value in all the functions, and it will be observed that the irrational part is

$$
= \pm \frac{2}{\left(x-x^{\prime}\right)^{2}} \sqrt{\begin{array}{l}
a-x \cdot b-x \cdot c-x \cdot d-x \cdot e-x \cdot f-x \\
a-x^{\prime} \cdot b-x^{\prime} \cdot c-x^{\prime} \cdot d-x^{\prime} \cdot e-x^{\prime} \cdot f-x^{\prime}
\end{array}}
$$

viz. this has the same value in all the functions.

The general property of the double 9 -functions is that the squares of the sixteen functions are proportional to constant multiples of the sixteen functions $[a]$, $[a b]$; but this theorem may be presented in a much more definite form, viz. we can determine, and 
that very simply, the actual expressions for the constant factors; and so we can enunciate the theorem as follows; the squares of the sixteen double 9 -functions are proportional to sixteen functions $-\{a\},+\{a b\}$; where, in a notation about to be explained,

$$
\{a\}=\sqrt{a}[a], \quad\{a b\}=\sqrt{a b}[a b] .
$$

Here in the radical $\sqrt{a}, a$ is to be considered as standing in the first place for the pentad bcdef, which is to be interpreted as a product of differences,

$$
=b c \cdot b d \cdot b e \cdot b f \cdot c d \cdot c e \cdot c f \cdot d e \cdot d f \cdot e f,
$$

(where $b c, b d$, etc., denote the differences $b-c, b-d$, etc.). Similarly, in the radical $\sqrt{a b}, a b$ is to be considered as standing in the first instance for the double triad abf.cde, which is to be interpreted as a product of differences, $=a b . a f . b f . c d . c e . d e$, (where $a b, a f$, etc., denote the differences $a-b, a-f$, etc.).

It is convenient to consider $a, b, c, d, e, f$ as denoting real magnitudes taken in decreasing order: in all the products bcdef, etc., and in each term abf or cde of a product $a b f . c d e$, the letters are to be written in alphabetical order; the differences $b c, b d$, etc., $a b, a f$, etc., which present themselves in the several products, are thus all of them positive; and the radicals, being all of them the roots of positive quantities, may themselves be taken to be positive.

We have to consider the values of the functions $[a],[a b]$, or $\{a\},\{a b\}$, in the case where the variables $x, x^{\prime}$ become equal to any two of the letters $a, b, c, d, e, f$; it is clearly the same thing whether we have for instance $x=b, x^{\prime}=c$, or $x=c, x^{\prime}=b$, etc.: we have therefore to consider for $x, x^{\prime}$ the fifteen values $a b, a c, \ldots, a f, \ldots, e f$; there is besides a sixteenth set of values $x, x^{\prime}$ each infinite, without any relation between the infinite values. have

Taking this ease first, $x, x^{\prime}$ each infinite, and in [ab], etc., the sign \pm to be + , we

$$
[a]=x x^{\prime}, \quad[a b]=\frac{4 x^{3} x^{\prime 3}}{\left(x-x^{\prime}\right)^{2}}
$$

or, attending only to the ratios of these values,

$$
[a]=1, \quad[a b]=\frac{4 x^{2} x^{\prime 2}}{\left(x-x^{\prime}\right)^{2}},
$$

where $\frac{4 x^{2} x^{\prime 2}}{\left(x-x^{\prime}\right)^{2}}$ is infinite, and the values may finally be written

whence also, for $x, x^{\prime}$ infinite,

$$
[a]=0, \quad[a b]=1 ;
$$

$$
\{a\}=0, \quad\{a b\}=\sqrt{a b},
$$

the radical $\sqrt{a b}$ being understood as before.

Suppose next that $x, x^{\prime}$ denote any two of the letters, for instance $a, b$; then two of the functions $[a]$ vanish, viz. these are $[a]$, $[b]$, but the remaining four functions acquire determinate values; and moreover four of the functions $[a b]$ vanish, viz. these are $[a b],[c d],[c e],[d e]$, for each of which the $x x^{\prime}$ letters $a, b$ occur in the same triad (the 
double triads for the four functions are, in fact, $a b f . c d e, c d f . a b e, c e f . a b d$, def.abc); but the other six functions $[a b]$, for which the letters $a, b$ occur in separate triads, acquire determinate values.

It is important to attend to the signs: for example, if $x, x^{\prime}=b, e$, we have

$$
\begin{gathered}
{[c]=c e \cdot c b, \quad=-b c \cdot c e} \\
{[c e]=\frac{1}{(b e)^{2}} a e \cdot b e \cdot d e, f b=-\begin{array}{c}
c b \cdot f b \\
a e \cdot d e
\end{array},=-\begin{array}{l}
b c \cdot b f \\
a e \cdot d e
\end{array} .}
\end{gathered}
$$

\begin{tabular}{|c|c|c|c|c|c|c|c|c|}
\hline$x$, & $x^{\prime}=\infty \infty$ & $a b$ & $a c$ & $a d$ & $a e$ & $a f$ & $b c$ & $b d$ \\
\hline$[a]$ & 0 & 0 & 0 & 0 & 0 & 0 & $+a b \cdot a c$ & $+a b \cdot a d$ \\
\hline$[b]$ & 0 & 0 & $-a b . b c$ & $-a b . b d$ & $-a b . b e$ & $-a b . b f$ & 0 & 0 \\
\hline$[c]$ & 0 & $+a c \cdot b c$ & 0 & $-a c \cdot c d$ & $-a c . c e$ & $-a c \cdot c f$ & 0 & $-b c \cdot c d$ \\
\hline$[d]$ & 0 & $+a d \cdot b d$ & $+a d \cdot c d$ & 0 & $-a d . d e$ & $-a d \cdot d f$ & $+b d \cdot c d$ & 0 \\
\hline$[e]$ & 0 & $+a e . b e$ & $+a e \cdot c e$ & $+a e \cdot d e$ & 0 & $-a e \cdot e f$ & $+b e . c e$ & $+b e \cdot d e$ \\
\hline$[f]$ & 0 & $+a f \cdot b f$ & $+a f \cdot c f$ & $+a f \cdot d f$ & $+a f \cdot e f$ & 0 & $+b f \cdot c f$ & $+b f . d f$ \\
\hline$[a b]$ & $+a b f . c d e$ & 0 & $+\begin{array}{r}a d \cdot a e \\
b c \cdot c f\end{array}$ & $+\begin{array}{l}a c \cdot a e \\
b d . d f\end{array}$ & $+\begin{array}{l}a c \cdot a d \\
b e \cdot e f\end{array}$ & 0 & $+\begin{array}{r}a c \cdot b d \\
b e \cdot c f\end{array}$ & $+\begin{array}{r}a d \cdot b c \\
b e \cdot d f\end{array}$ \\
\hline$[a c]$ & $+a c f . b d e$ & $-\begin{array}{l}a d \cdot a e \\
b c \cdot b f\end{array}$ & 0 & $+\begin{array}{l}a b . a e \\
c d . c f\end{array}$ & $+\begin{array}{l}a b \cdot a d \\
c e \cdot e f\end{array}$ & 0 & $+\begin{array}{l}a b . b f \\
c d . c e\end{array}$ & 0 \\
\hline$[a d]$ & $+a d f . b c e$ & $-\begin{array}{l}a c . a e \\
b d . b f\end{array}$ & $-\begin{array}{l}a b \cdot a e \\
c d \cdot c f\end{array}$ & 0 & $+\begin{array}{l}a^{7} \cdot a c \\
d e \cdot e f\end{array}$ & 0 & 0 & $-\begin{array}{l}a b \cdot b f \\
c d \cdot d e\end{array}$ \\
\hline$[a e]$ & $+a e f . b c d$ & $-\begin{array}{r}a c \cdot a e \\
b e \cdot b f\end{array}$ & $-\begin{array}{l}a b \cdot a d \\
c e \cdot c f\end{array}$ & $-\begin{array}{l}a b \cdot a c \\
d e . d f\end{array}$ & 0 & 0 & 0 & 0 \\
\hline$[b c]$ & $+b c f \cdot a d e$ & $-\begin{array}{l}a c \cdot a f \\
b d \cdot b e\end{array}$ & $-\begin{array}{l}a b \cdot a f \\
c d \cdot c e\end{array}$ & 0 & 0 & $-\begin{array}{l}a b . a c \\
d f . e f\end{array}$ & 0 & $-\begin{array}{l}a b \cdot b e \\
c d . d f\end{array}$ \\
\hline$[b d]$ & $+b d f$. ace & $-\begin{array}{l}a d . a f \\
b c . b e\end{array}$ & 0 & $+\begin{array}{l}a b \cdot a f \\
c d \cdot d e\end{array}$ & 0 & $-\begin{array}{l}a b . a d \\
c f \cdot e f\end{array}$ & $+\begin{array}{l}a b \cdot b e \\
c d . c f\end{array}$ & 0 \\
\hline$[b e]$ & $+b e f \cdot a c d$ & $\begin{array}{r}a e \cdot a f \\
b c \cdot b d\end{array}$ & 0 & 0 & $\begin{array}{r}a b \cdot a f \\
c e \cdot d e\end{array}$ & $-\begin{array}{c}a b \cdot a e \\
c f \cdot d f\end{array}$ & $+\begin{array}{l}a b \cdot b d \\
c e \cdot c f\end{array}$ & $+\begin{array}{l}a b . b c \\
d e . d f\end{array}$ \\
\hline$[c d]$ & $+c d f . a b e$ & 0 & $+\begin{array}{l}a d \cdot a f \\
b c \cdot c e\end{array}$ & $+\begin{array}{l}a c \cdot a f \\
b d \cdot d e\end{array}$ & 0 & $-\begin{array}{l}a c \cdot a d \\
b f \cdot e f\end{array}$ & $+\begin{array}{l}a c \cdot b d \\
b f \cdot c e\end{array}$ & $+\begin{array}{r}a d \cdot b c \\
b f \cdot d e\end{array}$ \\
\hline$[c e]$ & $+c e f \cdot a b d$ & 0 & $+\begin{array}{r}a e \cdot a f \\
b c \cdot c d\end{array}$ & 0 & $-\begin{array}{l}a c \cdot a f \\
b e \cdot d e\end{array}$ & $-\begin{array}{l}a c . a e \\
b f . d f\end{array}$ & $+\begin{array}{l}a c \cdot b e \\
b f \cdot c d\end{array}$ & 0 \\
\hline$[d e]$ & $+d e f . a b c$ & 0 & 0 & $-\begin{array}{l}a e \cdot a f \\
b c \cdot c d\end{array}$ & $-\begin{array}{l}a d \cdot a f \\
b e . c e\end{array}$ & $-\begin{array}{l}a d \cdot a e \\
b f \cdot c f\end{array}$ & 0 & $-\begin{array}{l}a d . b e \\
b f . c d\end{array}$ \\
\hline
\end{tabular}

TABle I. OF THE values of $[a],[a b]$, ETC., 
Here the symbols be, ce, etc., denote differences; $[c e]$ is the product of four differences: the arrangement in two lines is for convenience only.

We thus obtain the series of values of $[a]$, $[a b]$, etc., which although only required as subsidiary to the determination of the corresponding values of $\{a\},\{a b\}$, I nevertheless give in a table.

The signs are given as they were actually obtained, but as we are concerned only with the ratios of the functions, it is allowable to change all the signs in any

FOR THE SIXTEEN SPECIAI, VALUES OF $x, x^{\prime}$.

\begin{tabular}{|c|c|c|c|c|c|c|c|}
\hline be & $b f$ & $c d$. & $c e$ & $c f$ & $d e$ & $d f$ & $e f$ \\
\hline$+a b . a e$ & $+a b \cdot a f$ & $+a c . a d$ & $+a c \cdot a e$ & $+a c \cdot a f$ & $+a d . a e$ & $+a d \cdot a f$ & $+a e \cdot a f$ \\
\hline 0 & 0 & $+b c . b d$ & $+b c \cdot b e$ & $+b c \cdot b f$ & $+b d . b e$ & $+b d . b f$ & $+b e \cdot b f$ \\
\hline$-b c . c e$ & $-b c \cdot c f$ & 0 & 0 & 0 & $+c d \cdot c e$ & $+c d \cdot d f$ & $+c e \cdot c f$ \\
\hline$-b d . d e$ & $-b d . b f$ & 0 & $-c d \cdot d e$ & $-c d \cdot d f$ & 0 & 0 & $+d e \cdot d f$ \\
\hline 0 & - be eff & $+c e . d e$ & 0 & $-c e \cdot e f$ & 0 & $-d e . e f$ & 0 \\
\hline$+b f \cdot e f$ & 0 & $+c f \cdot d f$ & $+c f . e f$ & 0 & $+d f . e f$ & 0 & 0 \\
\hline$+\begin{array}{l}a c \cdot b c \\
b d \cdot e f\end{array}$ & 0 & 0 & 0 & $\begin{array}{r}a c \cdot b c \\
d f \cdot e f\end{array}$ & 0 & $-\begin{array}{l}a d \cdot b d \\
c f \cdot e f\end{array}$ & $-\begin{array}{l}a e . b e \\
c f . d f\end{array}$ \\
\hline 0 & $+\begin{array}{r}a b \cdot b c \\
d f \cdot e f\end{array}$ & $-\begin{array}{l}a d \cdot b c \\
c e \cdot d f\end{array}$ & $-\begin{array}{l}a e \cdot b c \\
c d \cdot e f\end{array}$ & 0 & 0 & $-\begin{array}{l}a d \cdot b f \\
c d . e f\end{array}$ & $-\begin{array}{l}a e \cdot b f \\
c e \cdot d f\end{array}$ \\
\hline 0 & $+\begin{array}{l}a b \cdot b d \\
c f \cdot e f\end{array}$ & $-\begin{array}{l}a c \cdot b d \\
c f \cdot d e\end{array}$ & 0 & $+\begin{array}{l}a c \cdot b f \\
c d \cdot e f\end{array}$ & $+\begin{array}{l}a e \cdot b d \\
c d \cdot e f\end{array}$ & 0 & $-\begin{array}{l}a e . b f \\
c f . d e\end{array}$ \\
\hline$+\begin{array}{l}a b \cdot b f \\
c e \cdot d e\end{array}$ & $+\begin{array}{l}a b \cdot b e \\
c f \cdot d f\end{array}$ & 0 & $+\begin{array}{r}a c \cdot b e \\
c f \cdot d e\end{array}$ & $+\begin{array}{l}a c \cdot b f \\
c e \cdot d f\end{array}$ & $+\begin{array}{l}a d \cdot b e \\
c e \cdot d f\end{array}$ & $+\begin{array}{l}a d . b f \\
c f . d e\end{array}$ & 0 \\
\hline $\begin{array}{r}a b \cdot b d \\
c e \cdot e f\end{array}$ & 0 & $-\begin{array}{l}a c \cdot b d \\
c e \cdot d f\end{array}$ & $\begin{array}{r}a c \cdot b e \\
c d \cdot e f\end{array}$ & 0 & 0 & $-\begin{array}{l}a f \cdot b d \\
c d \cdot e f\end{array}$ & $-\begin{array}{l}a f . b e \\
c e . d f\end{array}$ \\
\hline $\begin{array}{l}a d . b c \\
d e . e f\end{array}$ & 0 & $\begin{array}{r}a d \cdot b c \\
c f \cdot d e\end{array}$ & 0 & $+\begin{array}{l}a f \cdot b c \\
c d \cdot e f\end{array}$ & $+\begin{array}{l}a d \cdot b e \\
c d \cdot e f\end{array}$ & 0 & $\begin{array}{l}a f . b e \\
c f . d e\end{array}$ \\
\hline 0 & 0 & 0 & $+\begin{array}{r}a e \cdot b c \\
c f \cdot d e\end{array}$ & $+\begin{array}{l}a f \cdot b c \\
c e \cdot d f\end{array}$ & $+\begin{array}{l}a e \cdot b d \\
c e \cdot d f\end{array}$ & $+\begin{array}{l}a f \cdot b d \\
c f \cdot d e\end{array}$ & 0 \\
\hline 0 & $\begin{array}{l}a f \cdot b c \\
b d \cdot e f\end{array}$ & 0 & $+\begin{array}{r}a c \cdot b c \\
d e \cdot e f\end{array}$ & 0 & $+\begin{array}{l}a d \cdot b d \\
c e \cdot e f\end{array}$ & 0 & $-\begin{array}{l}a f \cdot b f \\
c e \cdot d e\end{array}$ \\
\hline $\begin{array}{l}a e \cdot b c \\
b f \cdot d e\end{array}$ & $\begin{array}{l}a f \cdot b c \\
b e \cdot d f\end{array}$ & $\begin{array}{c}a c \cdot b c \\
d e \cdot d f\end{array}$ & 0 & 0 & $+\begin{array}{l}a e \cdot b e \\
c d \cdot d f\end{array}$ & $+\begin{array}{l}a f \cdot b f \\
c d \cdot d e\end{array}$ & 0 \\
\hline$a e . b d$ & $a f . b d$ & $a d . b d$ & ae. be & $a f . b f$ & 0 & 0 & 0 \\
\hline
\end{tabular}

C. X. 
column: and it appears that there are four columns in each of which the signs are or can be made all +; whereas in each of the remaining twelve columns the signs are or can be made six of them + , the other four -.

Passing to the values of $\{a\},\{a b\}$, etc., we have for example, from the $a b$ column of the foregoing table,

$$
\begin{aligned}
& \{c\}=+\sqrt{c} \cdot a c \cdot b c, \\
& \{d\}=+\sqrt{d} \cdot a d \cdot b d, \\
& \text { ! } \\
& \{a c\}=-\sqrt{a c} \cdot \begin{array}{l}
a c \cdot a e \\
b c \cdot b f
\end{array},
\end{aligned}
$$

where (since the radicals are all positive) the signs are correct: substituting for the quantities under the radical signs their full values, and squaring the rational parts in order to bring them also under the radical signs, this is

$$
\begin{gathered}
\{c\}=+\sqrt{a b \cdot a d \cdot a e \cdot a f \cdot b d \cdot b e \cdot b f \cdot d e \cdot d f \cdot e f \cdot a c^{2} \cdot b c^{2}} \\
\{d\}=+\sqrt{a b \cdot a c \cdot a e \cdot a f \cdot b c \cdot b e \cdot b f \cdot c e \cdot c f \cdot e f \cdot a d^{2} \cdot b d^{2}} \\
\vdots \\
\{a c\}=-\sqrt{a c \cdot a f \cdot c f \cdot b d \cdot b e \cdot d e \cdot a c^{2} \cdot a e^{2} \cdot b c^{2} \cdot b f^{2}}
\end{gathered}
$$

where all the expressions of this (the $a b$-column) have a common factor,

$$
a c . a d \cdot a e \cdot a f . b c \cdot b d \cdot b e \cdot b f \text {. }
$$

Omitting this factor, we find

$$
\begin{gathered}
\{c\}=+\sqrt{a b \cdot a c \cdot b c \cdot d \cdot \cdot d f \cdot e f} \\
\{d\}=+\sqrt{a b \cdot a d \cdot b d \cdot c e \cdot c f \cdot e f} \\
\vdots \\
\{a c\}=-\sqrt{a d \cdot a e \cdot d e \cdot b c \cdot b f \cdot c f}
\end{gathered}
$$

viz. recurring to the foregoing condensed notation, this is

$$
\begin{gathered}
\{c\}=+\sqrt{d e} \\
\{d\}=+\sqrt{c e} \\
\vdots \\
\{a c\}=-\sqrt{b c}
\end{gathered}
$$

and, in fact, the terms in the several columns have only the ten values $\sqrt{a \bar{b}}, \sqrt{a c}$, etc. each with its proper sign. I repeat the meaning of the notation: $a b$ stands in the first instance for the double triad $a b f$.cde, and then this denotes a product of differences $a b . a f . b f . c d . c e . d e$. We have thus the following table in which I have in several cases changed the signs of entire columns. 


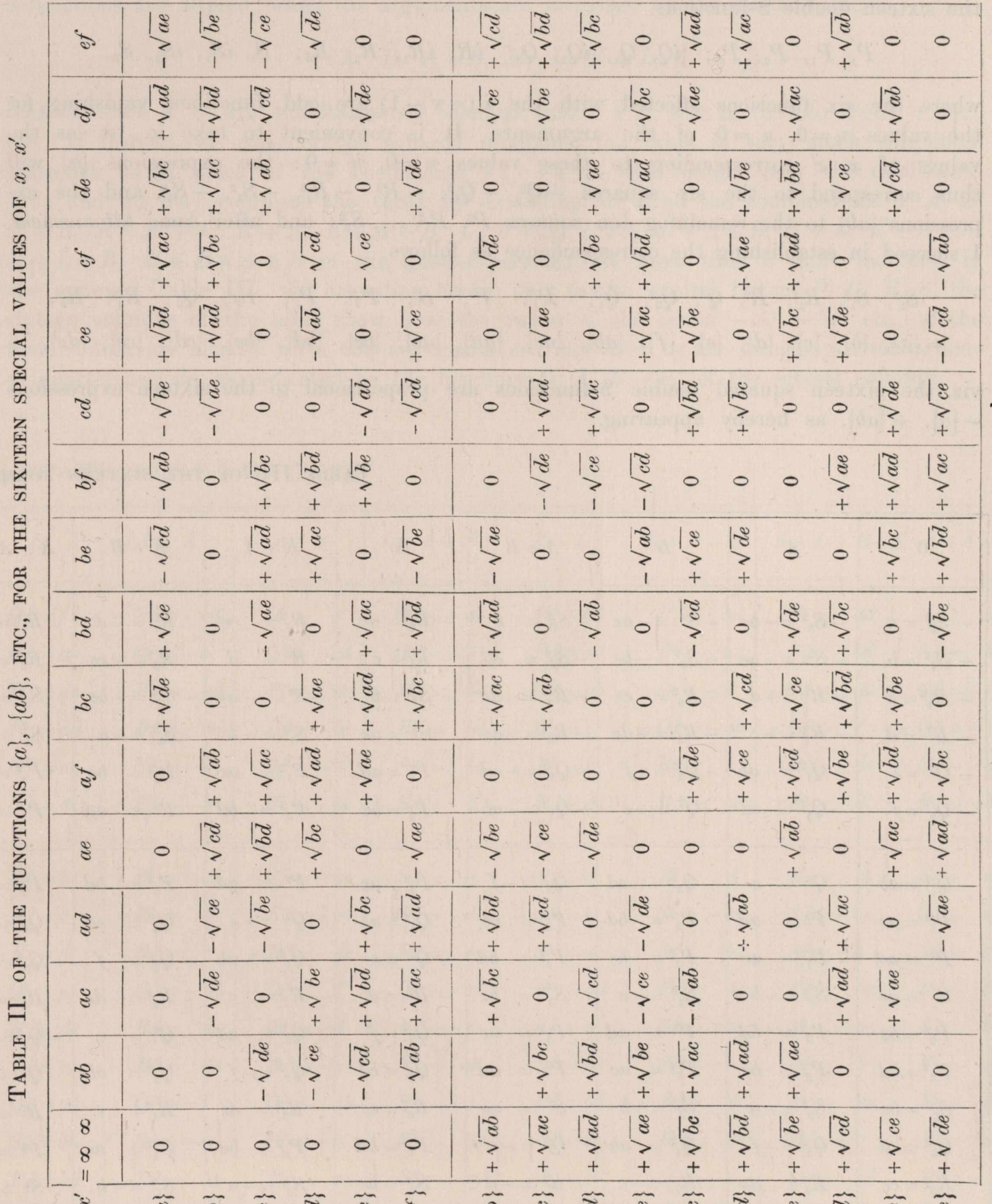

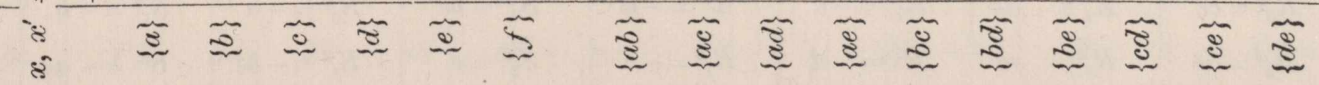


Referring now to Göpel's memoir, Crelle, t. xxxv. (1847), pp. 277-312, we have the sixteen double 9 -functions

$$
P, P_{1}, P_{2}, P_{3} ; i Q, Q_{1}, i Q_{2}, Q_{3} ; i R, i R_{1}, R_{2}, R_{3} ; S, i S_{1}, i S_{2}, S_{3},
$$

where the six functions affected with the $i(=\sqrt{-1})$ are odd functions, vanishing for the values $u=0, u^{\prime}=0$ of the arguments. It is convenient to take $\infty$, $\infty$ as the values of $x, x^{\prime}$ corresponding to these values $u=0, u^{\prime}=0$ : the expressions $\{a\}$ will thus correspond to the six squares $-Q^{2},-Q_{2}{ }^{2},-R^{2},-R_{1}{ }^{2},-S_{1}{ }^{2},-S_{2}{ }^{2}$, and the expressions $\{a b\}$ to the remaining ten squares $P^{2}, P_{1}^{2}, \ldots, S_{3}^{2}$; and after some tâtonnement, I succeed in establishing the correspondence as follows

$S_{2}^{2}, S_{1}^{2}, \quad R_{1}^{2}, R^{2}, Q^{2}, \quad Q_{2}^{2}, \quad Q_{1}^{2}, \quad P_{1}^{2}, \quad P^{2}, \quad S^{2}, \quad P_{2}^{2}, \quad P_{3}^{2}, \quad S_{3}^{2}, \quad Q_{3}^{2}, \quad R_{3}^{2}, \quad R_{2}{ }^{2}$,

$=\{a\},\{b\},\{c\},\{d\},\{e\},\{f\},\{a b\},\{a c\},\{a d\},\{a e\},\{b c\},\{b d\},\{b e\},\{c d\},\{c e\},\{d e\}$,

viz. the sixteen squared double 9 -functions are proportional to the sixteen expressions $-\{a\},+\{a b\}$, as hereby appearing.

TABLE III. OF THE SIXTEEN FORMS OF

\begin{tabular}{|c|c|c|c|c|c|c|c|}
\hline 0 & $A$ & $B$ & $A+B$ & K & $K+A$ & $K+B$ & $K+A+B$ \\
\hline$-S_{2}^{2}=a$ & $-S_{3}^{2}=-b e$ & $-S^{2}=-a e$ & $-S_{1}^{2}=b$ & $+R_{2}^{2}=d e$ & $R_{3}{ }^{2}=c e$ & $R^{2}=-d$ & $R_{1}^{2}=-c$ \\
\hline$-S_{1}^{2}=b$ & $-S^{2}=-a e$ & $-S_{3}^{2}=-b e$ & $-S_{2}^{2}=a$ & $-R_{1}^{2}=c$ & $-R^{2}=d$ & $-R_{3}{ }^{2}=-c e$ & $-R_{2}^{2}=-d e$ \\
\hline$-R_{1}^{2}=c$ & $-K^{2}=+d$ & $-R_{3}^{2}=-c e$ & $-R_{2}^{2}=-d e$ & $-S_{1}^{2}=b$ & $-S^{2}=-\boldsymbol{a} e$ & $-S_{3}^{2}=-b e$ & $-S_{2}^{2}=a$ \\
\hline$-R^{2}=d$ & $-R_{1}^{2}=+c$ & $-R_{2}{ }^{2}=-d e$ & $-R_{3}^{2}=-c e$ & $S^{2}=a e$ & $S_{1}^{2}=-b$ & $S_{2}^{\prime 2}=-a$ & $S_{3}{ }^{2}=b e$ \\
\hline$-Q^{2}=e$ & $-Q_{1}{ }^{2}=-a b$ & $-Q_{2}{ }^{2}=+f$ & $-Q_{3}{ }^{2}=-c d$ & $P^{2}=a d$ & $P_{1}^{2}=a c$ & $P_{2}^{2}=b c$ & $P_{3}^{2}=b d$ \\
\hline$-Q_{2}{ }^{2}=f$ & $-Q_{3}^{2}=-c d$ & $-Q^{2}=+e$ & $-Q_{1}^{2}=-a b$ & $P_{2}^{2}=b c$ & $P_{3}^{2}=b d$ & $P^{2}=a d$ & $P_{1}^{2}=a c$ \\
\hline$Q_{1}^{2}=a b$ & $Q^{2}=-e$ & $Q_{3}^{2}=c d$ & $Q_{2}{ }^{2}=-f$ & $P_{1}^{2}=a c$ & $P^{2}=a d$ & $P_{3}^{2}=b d$ & $P_{2}^{2}=b c$ \\
\hline$P_{1}^{2}=a c$ & $P^{2}=a d$ & $P_{3}^{2}=b d$ & $P_{2}^{2}=b c$ & $Q_{1}^{2}=a b$ & $Q^{2}=-e$ & $Q_{3}{ }^{2}=c d$ & $Q_{2}^{2}=-f$ \\
\hline$P^{2}=a d$ & $P_{1}^{2}=a c$. & $P_{2}^{2}=b c$ & $P_{3}^{2}=b d$ & $-Q^{2}=e$ & $-Q_{1}^{2}=-a b$ & $-Q_{2}{ }^{2}=f$ & $-Q_{3}{ }^{2}=-c d$ \\
\hline$S^{2}=a e$ & $S_{1}^{2}=-b$ & $S_{2}^{2}=-a$ & $S_{3}{ }^{2}=b e$ & $-R^{2}=d$ & $-R_{1}^{2}=c$ & $-R_{2}^{2}=-d e$ & $-R_{3}{ }^{2}=-c e$ \\
\hline$P_{2}^{2}=b c$ & $P_{3}^{2}=b d$ & $P^{2}=a d$ & $P_{1}^{2}=a c$ & $-Q_{2}{ }^{2}=f$ & $-Q_{3}{ }^{2}=-c d$ & $-Q^{2}=e$ & $-Q_{1}^{2}=-a b$ \\
\hline$P_{3}{ }^{2}=b d$ & $P_{2}^{2}=b c$ & $P_{1}^{2}=a c$ & $P^{2}=a d$ & $Q_{3}{ }^{2}=c d$ & $Q_{2}{ }^{2}=-f$ & $Q_{1}^{2}=a b$ & $Q^{2}=-e$ \\
\hline$S_{3}^{2}=b e$ & $S_{2}^{2}=-a$ & $S_{1}^{2}=-b$ & $S^{2}=a e$ & $R_{3}^{2}=c e$ & $R_{2}{ }^{2}=d e$ & $R_{1}^{2}=c$ & $R^{2}=-d$ \\
\hline$Q_{3}^{2}=c d$ & $Q_{2}{ }^{2}=-f$ & $Q_{1}^{2}=a b$ & $Q^{2}=-e$ & $P_{3}^{2}=b d$ & $P_{2}^{2}=b c$ & $P_{1}^{2}=a c$ & $P^{2}=a d$ \\
\hline$R_{3}^{2}=c e$ & $R_{2}^{2}=d e$ & $R_{1}=-c$ & $R^{2}=-d$ & $S_{3}^{2}=b e$ & $S_{2}^{2}=-a$ & $S_{1}^{2}=-b$ & $S^{2}=a e$ \\
\hline$R_{2}^{2}=d e$ & $R_{3}^{2}=c e$ & $R^{2}=-d$ & $R_{1}^{2}=-c$ & $-S_{2}^{2}=a$ & $-S_{3}^{2}=-b e$ & $-S^{2}=-a e$ & $-S_{1}^{2}=-b$ \\
\hline
\end{tabular}


We have, after Göpel (l.c. p. 283), a table showing how the ratios of the double 9 -functions are altered, when the arguments are increased by the quarter-periods

$$
A, B, A+B, K, L, K+L
$$

that is, when $u, u^{\prime}$ are simultaneously changed into $u+A, u^{\prime}+A^{\prime}$ or into $u+B, u^{\prime}+B^{\prime}$ etc. If instead, we consider the squared functions, the table is very much simplified, inasmuch as in place of the coefficients $\pm 1, \pm i$, it will contain only the coefficients \pm 1 : and we may complete the table by extending it to all the combinations $0, A, B$, $A+B, K, K+A, K+B, K+A+B, L, L+A, L+B, L+A+B, K+L, K+L+A$, $K+L+B, \quad K+L+A+B$ of the quarter-periods: we have thus a table included in the annexed Table III., viz. attending herein only to the capital letters $P, Q, R$, $S$, the sixteen columns of the table show how the ratios of the terms $-S_{2}^{2},-S_{1}^{2}$, etc., of the first column are altered when the arguments are increased by the foregoing combinations of quarter-periods, as indicated by the headings $0, A, B$, etc., of the several columns.

THE SQUARED DOUBLE T-FUNCTIONS.

\begin{tabular}{|c|c|c|c|c|c|c|c|}
\hline$L$ & $L+A$ & $L+B$ & $L+A+B$ & $K+L$ & $K+L+A$ & $K+L+B$ & $K+L+A+B$ \\
\hline$-Q_{2}{ }^{2}=f$ & $-Q_{3}{ }^{2}=-c d$ & $-Q^{2}=e$ & $-Q_{1}^{2}=-a b$ & $P_{2}^{2}=b c$ & $P_{3}{ }^{2}=b d$ & $P^{2}=a d$ & $P_{1}^{2}=a c$ \\
\hline$Q_{1}^{2}=a b$ & $Q^{2}=-e$ & $Q_{3}^{2}=c d$ & $Q_{2}^{2}=-f$ & $P_{1}^{2}=a c$ & $P^{2}=a d$ & $P_{3}^{2}=b d$ & $P_{2}^{2}=b c$ \\
\hline$P_{1}^{2}=a c$ & $P^{2}=a d$ & $P_{3}^{2}=b d$ & $P_{2}^{2}=b c$ & $Q_{1}^{2}=a b$ & $Q^{2}=-e$ & $Q_{3}{ }^{2}=c d$ & $Q_{2}{ }^{2}=-f$ \\
\hline$P^{2}=a d$ & $P_{1}^{2}=a c$ & $P_{2}^{2}=b c$ & $P_{3}^{2}=b d$ & $-Q^{2}=e$ & $-Q_{1}^{2}=-a b$ & $-Q_{2}{ }^{2}=f$ & $-Q_{3}{ }^{2}=-c d$ \\
\hline$S^{2}=a e$ & $S_{1}^{2}=-b$ & $S_{2}^{2}=-a$ & $S_{3}{ }^{2}=b e$ & $-R^{2}=d$ & $-R_{1}^{2}=c$ & $-R_{2}^{2}=-d e$ & $-R_{3}^{2}=-c e$ \\
\hline$-S_{2}^{2}=a$ & $-S_{3}{ }^{2}=-b e$ & $-S^{2}=-a e$ & $-S_{1}^{2}=b$ & $R_{2}^{2}=d e$ & $R_{3}^{2}=c e$ & $R^{2}=-d$ & $R_{1}^{2}=-c$ \\
\hline$-S_{1}^{2}=b$ & $-S^{2}=-a e$ & $-S_{3}^{2}=-b e$ & $-S_{2}^{2}=a$ & $-R_{1}^{2}=c$ & $-R^{2}=d$ & $-R_{3}^{2}=-c e$ & $-R_{2}^{2}=-d e$ \\
\hline$-R_{1}^{2}=c$ & $-R^{2}=d$ & $-R_{3}^{2}=-c e$ & $-R_{2}^{2}=-d e$ & $-S_{1}^{2}=b$ & $-S^{2}=-a e$ & $-S_{3}^{2}=-b e$ & $-S_{2}^{2}=a$ \\
\hline$-R^{2}=d$ & $-R_{1}^{2}=c$ & $-R_{2}^{2}=-d e$ & $-R_{3}^{2}=-c e$ & $S^{2}=a e$ & $S_{1}^{2}=b$ & $S_{2}^{2}=-a$ & $S_{3}{ }^{2}=b e$ \\
\hline$-Q^{2}=e$ & $-Q_{1}^{2}=-a b$ & $-Q_{2}{ }^{2}=f$ & $-Q_{3}^{2}=-c d$ & $P^{2}=a d$ & $P_{1}^{2}=a c$ & $P_{2}^{2}=b c$ & $P_{3}^{2}=b d$ \\
\hline$R_{2}^{2}=d e$ & $R_{3}{ }^{2}=c e$ & $R^{2}=-d$ & $R_{1}^{2}=-c$ & $-S_{2}^{2}=a$ & $-S_{3}^{2}=-b e$ & $-S^{2}=-a e$ & $-S_{1}^{2}=b$ \\
\hline$R_{3}{ }^{2}=c e$ & $R_{2}{ }^{2}=d e$ & $R_{1}^{2}=-c$ & $R^{2}=-d$ & $S_{3}{ }^{2}=b e$ & $S_{2}^{2}=-a$ & $S_{1}^{2}=-b$ & $S^{2}=a e$ \\
\hline$Q_{3}{ }^{2}=c d$ & $Q_{2}^{2}=-f$ & $Q_{1}^{2}=a b$ & $Q^{2}=-e$ & $P_{3}{ }^{2}=b d$ & $P_{2}^{2}=b c$ & $P_{1}^{2}=a c$ & $P^{2}=a d$ \\
\hline$S_{3}^{2}=b e$ & $S_{2}^{2}=-a$ & $S_{1}^{2}=-b$ & $S^{2}=a e$ & $R_{3}^{2}=c e$ & $R_{2}^{2}=d e$ & $R_{1}^{2}=-c$ & $R^{2}=-d$ \\
\hline$P_{3}^{2}=b d$ & $P_{2}^{2}=b c$ & $P_{1}^{2}=a c$ & $P^{2}=a d$ & $Q_{3}{ }^{2}=c d$ & $Q_{2}^{2}=-f$ & $Q_{1}^{2}=a b$ & $Q^{2}=-e$ \\
\hline$P_{2}^{2}=b c$ & $P_{3}{ }^{2}=b d$ & $P^{2}=a d$ & $P_{1}^{2}=a c$ & $-Q_{2}^{2}=f$ & $-Q_{3}{ }^{2}=-c d$ & $-Q^{2}=e$ & $-Q_{1}^{2}=-a b$ \\
\hline
\end{tabular}

$a f$

be

ae

$b f$

de

ce

$d f$

cf 
But I have also in the table inserted the values to which $-S_{2}{ }^{2},-S_{1}{ }^{2}$, etc., are respectively proportional, viz. the table runs $-S_{2}{ }^{2}=a,-S_{1}{ }^{2}=b$, etc., (read $-S_{2}{ }^{2}=\{a\}$, $-S_{1}{ }^{2}=\{b\}$, etc., the brackets \{\} having been for greater brevity omitted throughout the table), and where it is of course to be understood that $-S_{2}{ }^{2},-S_{1}{ }^{2}$, etc., are proportional only, not absolutely equal to $\{a\},\{b\}$, etc. And I have also at the foot of the several columns inserted suffixes $\infty \infty, a b, c d$, etc., which refer to the columns of Table II.

Comparing the first with any other column of the table, for instance with the second column, the two columns respectively signify that

$$
\begin{array}{c|c}
-S_{2}^{2}(u)=\{a\}, & -S_{2}^{2}(u+A)=-\{b e\}, \\
-S_{1}{ }^{2}(u)=\{b\}, & -S_{1}{ }^{2}(u+A)=-\{a e\}, \\
\vdots & \vdots \\
Q_{1}{ }^{2}(u)=\{a b\}, & Q_{1}{ }^{2}(u+A)=-\{e\}, \\
\vdots & \vdots
\end{array}
$$

where, as before, the sign $=$ means only that the terms are proportional; $u$ is written for shortness instead of $\left(u, u^{\prime}\right)$, and so $u+A$ for $\left(u+A, u^{\prime}+A^{\prime}\right)$, etc.: the variables in the functions $\{a\},\{b e\}$, etc. are in each case $x, x^{\prime}$. But if in the second column we write $u-A$ for $A$, then the variables $x, x^{\prime}$ will be changed into new variables $y, y^{\prime}$, or the meaning will be

$$
\begin{array}{c||r}
x, x^{\prime} & y, y^{\prime} \\
-S_{2}{ }^{2}(u)=\{a\}, & -S_{2}{ }^{2}(u)=-\{b e\}, \\
-S_{1}{ }^{2}(u)=\{b\}, & -S_{1}{ }^{2}(u)=-\{a e\}, \\
\vdots & \vdots \\
Q_{1}{ }^{2}(u)=\{a b\}, & Q_{1}{ }^{2}(u)=-\{e\}, \\
\vdots & \vdots
\end{array}
$$

so that, omitting from the table the terms which cuntain the capital letters $P, Q, R, S$, except only the outside left-hand column $-S_{2}{ }^{2},-S_{1}{ }^{2}$, etc., the table indicates that these functions $-S_{2}{ }^{2},-S_{1}{ }^{2}$, etc., are proportional to the functions $\{a\},\{b\}$, etc., of $x$, $x^{\prime}$ given in the first column; also to the functions $-\{b e\},-\left\{a e^{\prime}\right.$, etc., of $y, y^{\prime}$ given in the second column; also to the functions $-\{a e\},-\{b e\}$, etc., of $z, z^{\prime}$ given in the third column; and so on, with a different pair of variables in each of the 16 columns.

Thus comparing any two columns, for instance the first and second, it appears that we can have simultaneously

$$
\begin{gathered}
x, x^{\prime} \quad y, y^{\prime} \\
\{a\}=-\{b e\} \\
\{b\}=-\{a e\} \\
\vdots \\
\{a b\}=-\{e\} \\
\vdots
\end{gathered}
$$

(fifteen equations, since the meaning is that the terms are only proportional, not absolutely equal), equivalent to two equations serving to determine $x$ and $x^{\prime}$ in terms of $y$ and $y^{\prime}$, 
or conversely $y$ and $y^{\prime}$ in terms of $x$ and $x^{\prime}$. The functions in each column form in fact 16 sixes, such that any four belonging to the same six are linearly connected; and in any such linear relation between four functions in the left-hand column, substituting for these their values as functions in the right-hand column, we have the corresponding relations between four functions out of a set of six belonging to the right-hand column, or we have an identity $0=0$. I will presently verify this in a particular case.

If in any column we give to the variables the values $\infty, \infty$ we obtain for the terms in the column the values which the terms of the first column assume on giving to $x, x^{\prime}$ the values shown at the foot of the column in question; thus, in the second column giving to the variables the values $\infty, \infty$, the column becomes

$$
-\sqrt{b e},-\sqrt{a e}, 0,0,-\sqrt{a b},-\sqrt{c d}, 0, \sqrt{a d}, \sqrt{a c}, 0, \sqrt{b d}, \sqrt{b c}, 0,0, \sqrt{d e}, \sqrt{c e}
$$

which is, in fact, the $c d$-column of Table II.: this is of course as it should be, for the values in question are those of the functions $-S_{2}{ }^{2},-S_{1}^{2}$, etc., on writing therein

The formulæ show that

$$
x, x^{\prime}=c, d .
$$

$\sqrt{a b}, \quad \sqrt{a c}, \quad \sqrt{a d}, \quad \sqrt{a e}, \quad \sqrt{b c}, \quad \sqrt{b d}, \quad \sqrt{b e}, \quad \sqrt{c d}, \quad \sqrt{c e}, \quad \sqrt{d e}$,

are, in fact, proportional to

$$
k_{1}^{2}, \quad \varpi_{1}^{2}, \quad \varpi^{2}, \quad \sigma^{2}, \quad \varpi_{2}{ }^{2}, \quad \varpi_{3}{ }^{2}, \quad \sigma_{3}{ }^{2}, \quad k_{3}{ }^{2}, \quad \rho_{3}{ }^{2}, \quad \rho_{2}{ }^{2},
$$

$\left(k_{1}, k_{2}, \ldots\right.$ are Göpel's $\left.k^{\prime}, k^{\prime \prime}, \ldots\right)$. This gives rise to a remarkable theorem, for the ten squares are functions of only four quantities $\alpha, \beta, \gamma, \delta$ (Göpel's $t, u, v, w$ ). For greater clearness, I introduce single letters $A, B, \ldots, J$ and write

$$
\begin{aligned}
& A=a b c \cdot d e f=(\sqrt{d e})^{2}=\rho_{2}{ }^{4},=\left(\alpha^{2}-\beta^{2}+\gamma^{2}-\delta^{2}\right)^{2}, \\
& B=a b d \cdot c e f=(\sqrt{c e})^{2}=\rho_{3}{ }^{4}, \quad=4(\alpha \gamma+\beta \delta)^{2}, \\
& C=a b e \cdot c d f=(\sqrt{c d})^{2}=k_{3}{ }^{4}, \quad=4(\alpha \delta+\beta \gamma)^{2}, \\
& D=a b f \cdot c d e=(\sqrt{a b})^{2}=k_{1}{ }^{4}, \quad=\left(\alpha^{2}-\beta^{2}-\gamma^{2}+\delta^{2}\right)^{2}, \\
& E=a c d \cdot b e f=(\sqrt{b e})^{2}=\sigma_{3}{ }^{4}, \quad=4(\alpha \beta+\gamma \delta)^{2}, \\
& F=a c e \cdot b d f=(\sqrt{b d})^{2}=\sigma_{3}{ }^{4}, \quad=\left(\alpha^{2}+\beta^{2}+\gamma^{2}+\delta^{2}\right)^{2}, \\
& G=a c f \cdot b d e=(\sqrt{a c})^{2}=\sigma_{1}{ }^{4}, \quad=4(\alpha \delta-\beta \gamma)^{2}, \\
& H=a d e \cdot b c f=(\sqrt{b c})^{2}=\sigma_{2}{ }^{4},=4(\alpha \gamma-\beta \delta)^{2}, \\
& I=a d f \cdot b c e=(\sqrt{a d})^{2}=\sigma^{4}, \quad=4(\alpha \beta-\gamma \delta)^{2}, \\
& J=a e f \cdot b d c=(\sqrt{a e})^{2}=\sigma^{4}, \quad=\left(\alpha^{2}+\beta^{2}-\gamma^{2}-\delta^{2}\right)^{2} ;
\end{aligned}
$$

viz. it has to be shown that $A, B, \ldots, J$, considered as given functions of the six letters $a, b, c, d, e, f$, are really functions of four quantities $\alpha, \beta, \gamma, \delta$; or, what is the same thing, that $A, B, \ldots, J$, considered as functions of $a, b, c, d, e, f$ satisfy all those relations which they satisfy when considered as given functions of $\alpha, \beta, \gamma, \delta$. 
Now considering them as given functions of $\alpha, \beta, \gamma, \delta$, they ought to satisfy six relations; and inasmuch as, so considered, they are, in fact, linear functions of

$$
\alpha^{4}+\beta^{4}+\gamma^{4}+\delta^{4}, \quad \alpha^{2} \beta^{2}+\gamma^{2} \delta^{2}, \quad \alpha^{2} \gamma^{2}+\beta^{2} \delta^{2}, \quad \alpha^{2} \delta^{2}+\beta^{2} \gamma^{2}, \quad \alpha \beta \gamma \delta,
$$

five of these relations will be linear: there is a sixth non-linear relation, expressible in a variety of different forms, one of them, as is easily verified, being

$$
\sqrt{A J} \pm \sqrt{C G} \pm \sqrt{D F}=0 .
$$

Now considering $A, B, \ldots, J$ as given functions of $a, b, c, d, e, f$, there exist between them linear relations which may be obtained by the consideration of identities of the form

$$
\left|\begin{array}{l}
\text { abcd } \\
\text { abcdef }
\end{array}\right|=0,
$$

where the left-hand side is used for shortness to denote the determinant

$$
\left|\begin{array}{llllll}
1, & 1, & 1, & 1 \\
a, & b, & c, & d & & \\
a^{2}, & b^{2}, & c^{2}, & d^{2} & & \\
1, & 1, & 1, & 1, & 1, & 1 \\
a, & b, & c, & d, & e, & f \\
a^{2}, & b^{2}, & c^{2}, & d^{2}, & e^{2}, & f^{2}
\end{array}\right|=0 .
$$

We thus obtain between them a system of fifteen linear relations, which present themselves in the form

$$
\begin{array}{rrr}
(1) & A-J+E-B & =0 \\
(2) & -A-I+F-C & =0 \\
(3) & A-H+G-D & =0 \\
(4) & -B-G+H+C & =0 \\
(5) & B-F+I+D & =0 \\
(6) & C-E+J-D & =0 \\
(7) & -E-D-H+E & =0 \\
(8) & E-C-I+G & =0 \\
(9) & F-B-J-G & =0 \\
\text { (10) } & H-A+J-I & =0 \\
\text { (11) } & -J+D-G+I & =0 \\
\text { (12) } & J+C-F+H & =0 \\
\text { (13) } & I+B-E-H & =0 \\
\text { (14) } & G+A+E-F & =0 \\
\text { (15) } & D-A+B-C & =0
\end{array}
$$


and these are all included in the equations (10), (4), (12), (15), (6), which serve to express $G, B, E, F, I$ in terms of $D, H, C, A, J$, i.e. $a c, c e, e b, b d, d a$ in terms of $a b, b c, c d$, de, $e a$, if for the moment we write $G=a c$, etc. But the five linear relations in question are, it is at once seen, satisfied by $A, B, \ldots, J$ considered as given functions of $\alpha, \beta, \gamma, \delta$.

The equation $\sqrt{A J} \pm \sqrt{D F} \pm \sqrt{C G}=0$, substituting for $A, B, \ldots, J$ their values in terms of $a, b, c, d, e, f$, becomes

$$
\sqrt{a b c . d e f . a e f . b c d} \pm \sqrt{a b f . c d e . a c e \cdot b d f} \pm \sqrt{a b e \cdot c d f . a c f . b d e}=0
$$

which (omitting common factors) becomes $\sqrt{b c^{2} \cdot e f^{2}} \pm \sqrt{b f^{2} \cdot c e^{2}} \pm \sqrt{b e^{2} \cdot c f^{2}}=0$; or, taking the proper signs, this is the identity $b c . e f+b e \cdot f c+b f . c e=0$.

It is to be noticed that

$$
\begin{array}{rrr}
\delta^{2}+\alpha^{2}-\beta^{2}-\gamma^{2}, & 2(\alpha \beta-\gamma \delta), & 2(\gamma \alpha+\beta \delta), \\
2(\alpha \beta+\gamma \delta), & \delta^{2}+\beta^{2}-\gamma^{2}-\alpha^{2}, & 2(\beta \gamma-\alpha \delta), \\
2(\gamma \alpha-\beta \delta), & 2(\beta \gamma+\alpha \delta), & \delta^{2}+\gamma^{2}-\alpha^{2}-\beta^{2},
\end{array}
$$

each divided by $\delta^{2}+\alpha^{2}+\beta^{2}+\gamma^{2}$, form a system of coefficients in the transformation between two sets of rectangular coordinates. We have therefore

$$
\begin{array}{lll}
\sqrt{a b}, & \sqrt{a d}, & \sqrt{c e}, \\
\sqrt{b e}, & \sqrt{d e}, & \sqrt{a c}, \\
\sqrt{b c}, & \sqrt{c d}, & \sqrt{a e},
\end{array}
$$

each divided by $\sqrt{b d}$ and the several terms taken with proper signs, as a system of coefficients in the transformation between two sets of rectangular axes: a result which seems to be the same as that obtained by Hesse in the Memoir, "TransformationsFormeln für rechtwinklige Raum-Coordinaten"; Crelle, t. LXIII. (1864), pp. 247-251.

The composition of the last mentioned system of functions is better seen by writing them under the fuller form $\sqrt{ } a b f$.cde, etc.; viz. omitting the radical signs, the terms are

$$
\begin{array}{lll}
a b f . c d e, & a d f . b c e, & a b d . c e f, \\
b e f . a c d, & d e f . a b c, & a c f . b d e, \\
b c f . a d e, & c d f . a b e, & a e f . b c d,
\end{array}
$$

each divided by $b d f$.ace; or, in an easily understood algorithm, the terms are

each divided by $b d f$. ace.

$$
\begin{array}{l|lll}
\multicolumn{1}{c|}{} & b f . d & d f . b & b d . f \\
\cline { 2 - 4 } a . c e & b f . d & d f . b & b d . f \\
e . a c & b f . d & d f . b & b d . f \\
c . a e & b f . d & d f . b & b d . f
\end{array}
$$

c. $\mathrm{X}$. 
Reverting to the before-mentioned comparison of the first and second columns of "Table III., four of the equations are

$$
\begin{aligned}
& x, x^{\prime} \quad y, y^{\prime} \quad x, x^{\prime} \quad y, y^{\prime} \\
& \{c\}=\{d\}, \quad \text { that is, } \sqrt{c}[c]=\sqrt{d}[d], \\
& \{d\}=\{c\}, \quad \text { that is, } \sqrt{d}[d]=\sqrt{c}[c] \text {, } \\
& \{e\}=-\{a b\}, \quad \text { that is, } \sqrt{e}[e]=-\sqrt{a b}[a b], \\
& \{f\}=-\{c d\}, \quad \text { that is, } \sqrt{f}[f]=-\sqrt{c d}[c d] \text {; }
\end{aligned}
$$

viz. the four terms on the left-hand side are not absolutely equal, but only proportional, to those on the right-hand side. Substituting for $\sqrt{c}, \sqrt{d}$, etc., their values, and introducing on the right-hand side the factor

the equations become

$$
\sqrt{a c \cdot b c \cdot c e \cdot c f \cdot a d \cdot b d \cdot d e \cdot d f},
$$

$$
\begin{array}{lc}
x x^{\prime} & y y^{\prime} \\
{[c]=} & a c . b c \cdot c e \cdot e f[d], \\
{[d]=} & a d . b d . d e \cdot d f[c], \\
{[e]=-} & c e \cdot d e[a b], \\
{[f]=-} & c f \cdot d f[c d] .
\end{array}
$$

The functions on the left-hand satisfy the identity

$$
\operatorname{def}[c]-e f c[d]+f c d[e]-c d e[f]=0,
$$

or, as this may also be written,

$$
\operatorname{def}[c]-\operatorname{cef}[d]+c d f[e]-c \operatorname{de}[f]=0 .
$$

Hence substituting the right-hand values, the whole equation divides by $c e . d e . c f . d f$; omitting this factor, it becomes

$$
e f . a c . b c[d]-e f . a d . b d[c]-c d\{[a b]-[c d]\}=0,
$$

where the variables are $y, y^{\prime}$ : it is to be shown that this is in fact an identity, and (as it is thus immaterial what the variables are) I change them into $x, x^{\prime}$.

We have

$$
\begin{aligned}
a c . b c[d]-a d . b d[c] & =(a-c)(b-c)(d-x)\left(d-x^{\prime}\right) \\
& -(a-d)(b-d)(c-x)\left(c-x^{\prime}\right) \\
= & (c-d)\left|\begin{array}{lll}
1, & x+x^{\prime}, & x x^{\prime} \\
1, & a+b, & a b \\
1, & c+d, & c d
\end{array}\right| \\
= & c d\left[x x^{\prime} a b c d\right],
\end{aligned}
$$

suppose. 
We have moreover

$$
\begin{aligned}
{[a b]-[c d] } & =\frac{1}{\left(x-x^{\prime}\right)^{2}}\left\{\begin{array}{r}
a b f \cdot c^{\prime} d^{\prime} e^{\prime}+a^{\prime} b^{\prime} f^{\prime} \cdot c d e \\
-c d f \cdot a^{\prime} b^{\prime} e^{\prime}-c^{\prime} d^{\prime} f^{\prime} \cdot a b e
\end{array}\right\} \\
& =\frac{1}{\left(x-x^{\prime}\right)^{2}}\left(a b c^{\prime} d^{\prime}-a^{\prime} b^{\prime} c d\right)\left(e^{\prime} f-e f^{\prime}\right)
\end{aligned}
$$

where for the moment $a, b, a^{\prime}$, etc., are written to denote $a-x, b-x, a-x^{\prime}$, etc.; we have then

and

$$
\begin{aligned}
e^{\prime} f-e f^{\prime} & =\left(e-x^{\prime}\right)(f-x)-(e-x)\left(f-x^{\prime}\right) \\
& =-(e-f)\left(x-x^{\prime}\right)=-e f\left(x-x^{\prime}\right)
\end{aligned}
$$

$$
\begin{aligned}
a b c^{\prime} d^{\prime}-a^{\prime} b^{\prime} c d & =(a-x)(b-x)\left(c-x^{\prime}\right)\left(d-x^{\prime}\right)=-\left(x-x^{\prime}\right)\left|\begin{array}{ccc}
1, & x+x^{\prime}, & x x^{\prime} \\
1, & a+b, & a b \\
1, & c+d, & c d
\end{array}\right| \\
-\left(a-x^{\prime}\right)\left(b-x^{\prime}\right)(c-x)(d-x) & =-\left(x-x^{\prime}\right)\left[x x^{\prime} a b c d\right] .
\end{aligned}
$$

Hence $[a b]-[c d]=e f\left[x x^{\prime} a b c d\right]$, and the equation to be verified becomes

$$
(e f . c d-c d . e f)\left[x x^{\prime} a b c d\right]=0,
$$

viz. this is, in fact, an identity.

Cambridge, 14 March, 1877. 